\title{
Effects of Maternal Prenatal Smoking and Birth Outcomes Extending into the Normal Range on Academic Performance in Fourth Grade in North Carolina, USA
}

\author{
Rebecca Anthopolos, ${ }^{a}$ Sharon E. Edwards, ${ }^{a}$ Marie Lynn Mirandaa, \\ ${ }^{a}$ School of Natural Resources and Environment \\ ${ }^{b}$ Department of Pediatrics, University of Michigan, Ann Arbor, MI
}

\begin{abstract}
Background: Research has documented the adverse relationship of child cognitive development with maternal prenatal smoking and poor birth outcomes. The potential, however, for maternal prenatal smoking to modify the association between birth outcomes and cognitive development is unclear.

Methods: We linked statewide North Carolina birth data for non-Hispanic white and non-Hispanic black children to end-of-grade test scores in reading and mathematics at fourth grade $(n=65677)$. We fit race-stratified multilevel models of test scores regressed on maternal smoking, birth outcomes (as measured by continuous and categorical gestational age and birthweight percentile for gestational age), and their interaction, controlling for maternal- and child-level socio-economic factors.

Results: Smoking was consistently associated with decrements in test scores, and better birth outcomes were associated with improvements in test scores, even in clinically normal ranges. Test scores increased quadratically with improving birth outcomes among smoking and non-smoking mothers. Among non-Hispanic white children, the magnitude of the association between gestational age and test scores was larger for children whose mothers smoked during pregnancy compared with the non-smoking group. However, among non-Hispanic black children, birth outcomes did not appear to interact with maternal smoking on test scores.

Conclusions: Maternal prenatal smoking may interact with birth outcomes on reading and mathematics test scores, particularly among non-Hispanic white children. Improvements in birth outcomes, even within the clinically normal range, may be associated with improved academic performance. Pregnancy-related exposures and events exert a significant and long-term impact on cognitive development.
\end{abstract}

Keywords: achievement, cognition, learning disorders, preterm birth, small for gestational age.

A large body of literature provides evidence that extreme prematurity is associated with significant decrements in children's cognitive development, resulting in long-term impacts on learning and academic performance. ${ }^{1-5}$ While numerous studies have focused on extremely preterm infants, the recent rise in late preterm birth has increased interest in exploring whether decrements in cognitive development occur across a wider spectrum of birth outcomes, including among late preterm births (34-36 weeks gestational age), moderately low birthweight births (1500-2499 g), and even term and normal birthweight births. Decrements in measures of cognitive development have

\section{Correspondence:}

Marie Lynn Miranda, School of Natural Resources and Environment and Department of Pediatrics, University of Michigan, 440 Church Street, Ann Arbor, MI 48109, USA.

E-mail: mlmirand@umich.edu been associated with decreasing birthweights within the normal range $(\geq 2500 \mathrm{~g})$, $^{5-7}$ and with decreasing gestational ages within the late preterm and term ranges. ${ }^{8-11}$

Maternal prenatal smoking has been associated with impaired fetal growth and adverse birth outcomes, ${ }^{12,13}$ and although the evidence is less clear, maternal smoking during pregnancy may also be associated with impairments in children's cognitive development. ${ }^{12}$ Several studies have shown a negative association between maternal smoking during pregnancy and performance on measures of cognitive development, including IQ and school achievement. ${ }^{6,12,14-18}$ However, the relationship between a child's IQ and maternal prenatal smoking has been called into question by recent studies that found the relationship to be attenuated when controlling for maternal and familial characteristics. ${ }^{19-21}$ 
While previous studies have focused on the twoway relationships between smoking and cognitive development, perinatal outcomes and cognitive development, or smoking and perinatal outcomes, few studies have explored how both poor birth outcomes and maternal prenatal smoking may interact to influence academic achievement. ${ }^{6,20}$ In this analysis, we extend the existing literature by bringing these three relationships together in order to explore whether maternal prenatal smoking modifies the association between birth outcomes and test scores. Using a population-level dataset for the State of North Carolina (NC), we examine whether birth outcomes and maternal prenatal smoking are associated with cognitive development, as indicated by end-of-grade (EOG) test scores in reading and mathematics. We investigate effect modification at clinically defined thresholds of gestational age (very preterm, late preterm, and term births) and across the full spectrum of continuous gestational ages, along with corresponding analysis investigating fetal growth as measured by birthweight percentile for gestational age and the related clinical indicator of small for gestational age.

\section{Methods}

\section{Data sources}

Data for this study were drawn from two sources: the NC Detailed Birth Record (NCDBR) and the NC Education Research Data Center (NCERDC). The NCDBR provides data on all documented live births occurring in NC, including information on maternal demographics, maternal and infant health, and maternal obstetrics history. In validation studies, birth certificate data have been shown to be accurate, particularly for demographic and birth outcome variables. ${ }^{22,23}$ The NCERDC maintains a database of standardised test results for public school children in NC. Known as EOG tests, standardised tests in NC measure student performance on grade-based goals, objectives, and competencies, and are mandatory for all children in grades $3-8 .{ }^{24}$ The multiple choice reading test covers cognition, interpretation, critical stance, and connections. ${ }^{25}$ The multiple choice mathematics test assesses number sense, numeration, and numerical operations; spatial sense, measurement, and geometry; patterns, relationships, and functions; and data, probability, and statistics. ${ }^{24}$ This study was performed in accord- ance with a protocol approved by the University of Michigan Institutional Review Board.

\section{Linking datasets}

We linked birth records with educational data for NC children in fourth grade during the 2003-04 school year. Records were linked through an iterative deterministic process employing various combinations of identifying variables (first and last name, date of birth, and county of residence) and requirements on the stringency of the match strength. This process allowed for records to be linked despite misspellings, typos, and abbreviations. County of residence was only included in the first linkage iteration to allow for residential mobility. Of 105953 unique children taking fourth grade EOG tests during the 2003-04 school year, $72208(68 \%)$ were successfully matched to a record in the NCDBR. Of the 98301 children born in NC between September 1993 and August 1994, the timing of births for most $\mathrm{NC}$ fourth grade students in the 2003-04 school year, 52206 (53\%) were linked to a record in the EOG data.

\section{Study sample}

We restricted to children born to self-reported nonHispanic white (NHW) and non-Hispanic black (NHB) mothers (excluded 3481 records) because of small cell numbers in the Hispanic, Asian, and other race/ethnicity categories, as well as complications in interpreting test scores among young children for whom English is their second language. Mothers included in our analysis were between the ages of 15 and 44 years (excluded 359 records), and had no more than three previous live births (excluded 1646 records). Children weighed at least $400 \mathrm{~g}$ (excluded three records), had a gestational age at delivery between 24 and 42 weeks (excluded 98 records), and had no congenital anomalies (excluded 751 records). We also removed 44 records for which child sex information did not match between the datasets and 149 records with missing values for key covariates. Our final dataset included 65677 children, of whom 64302 and 64486 had complete reading and mathematics EOG test scores, respectively.

\section{Statistical analysis}

We used continuous reading and mathematics EOG test scores as dependent variables. Our exposures 
were gestational age at delivery in completed weeks and birthweight percentile for gestational age, and maternal smoking status. Birthweight percentile for gestational age serves as a measure of fetal growth, and was determined by comparing the birthweight for each birth in our study population with a national sexspecific reference distribution. ${ }^{26}$ We examined these birth outcome exposures as both continuous and categorical measures. In the categorical analysis, gestational age was grouped as $<34$ weeks (very preterm birth), 35-36 weeks (late preterm birth), and $\geq 37$ weeks (term birth; reference level), while birthweight percentile for gestational age was categorised into an indicator of small for gestational age $(\leq 10$ th percentile). Maternal smoking status was derived from selfreported smoking during pregnancy, as reported in the NCDBR.

The analysis controlled for potentially confounding maternal and child-level factors. Maternal characteristics collected at the time of delivery included age in years, categorised as 15-19, 20-24, 25-29 (reference level), 30-34, 35-39, and 40-44; educational attainment, categorised as less than high school $(<12$ th grade), completed high school (12th grade; reference level), some college (13-15 years of education), and completed college ( $\geq 16$ years of education); and marital status. We adjusted for child male sex and participation in the school free or reduced lunch programme.

Preliminary exploratory analysis revealed differential covariate distributions by race group (see Table 1). To accommodate racial heterogeneity in our analysis, we fit race-stratified models of EOG test scores regressed on birth outcomes and maternal smoking status, adjusting for maternal and child factors, separately by subject matter and birth outcome. We conducted multilevel analysis, nesting children within their schools and school districts to account for potential unobserved heterogeneity in school and districtlevel quality and socio-economic context. To investigate whether the relationship between EOG test score and each birth outcome differed by maternal smoking, we included an interaction term between the two exposures. In models with continuously measured birth outcomes, we included a quadratic birth outcome term to allow for non-linear relationships with test scores.

We used model coefficients from the specifications with categorical birth outcome exposures to estimate the marginal effect of maternal prenatal smoking by birth outcome categories. For continuously measured birth outcomes, we predicted mean test scores according to the spectrum of birth outcome values, by maternal smoking status. Remaining covariates were held at their reference values, and standard errors were calculated with the delta method. ${ }^{27}$ All statistical analyses were performed in R 2.15.1 (The R Foundation for Statistical Computing 2012, Vienna, Austria).

\section{Results}

Compared with NHW children, NHB children had lower mean reading and mathematics scores (Table 1). While mean gestational age was equal between race groups, NHB children were more likely to be born before 37 weeks than NHW children (13\% vs. $8 \%)$. Mean birthweight percentile for gestational age was 51 for NHW children compared with 39 for NHB children, with $17 \%$ of NHB children classified as small for gestational age compared with only $9 \%$ of NHW children. Over $20 \%$ of NHW mothers reported smoking during pregnancy, compared with $12 \%$ of NHB mothers.

We focus the modelling results presented herein on the primary exposures of birth outcomes and maternal prenatal smoking status, and their interactions on test scores. We refer the reader to the online supporting information for the full set of estimated coefficients and variance terms for each model. A brief summary of covariate behaviour is provided following the main findings.

\section{Gestational age}

The marginal effects of maternal prenatal smoking by gestational age category, including $<34$ weeks (very preterm), 34-36 weeks (late preterm), and $\geq 37$ weeks (term), are presented in Table 2 . In the NHW reading analysis, children born to mothers who reported smoking during pregnancy had lower mean reading scores for each gestational category than their counterparts in the non-smoking category. For example, NHW children born at $<34$ weeks to smokers had average test scores 1.92 points lower than those of children born at $<34$ weeks to non-smoking mothers [marginal effect $=-1.92,95 \%$ confidence interval $(\mathrm{CI})$ $-3.03,-0.81]$. The magnitude of the marginal effect of smoking during pregnancy diminished with increasing gestational age category (see Table 2). In the NHB reading analysis, $\mathrm{NHB}$ children born at $<34$ weeks to 
Table 1. Summary statistics of reading and mathematics end-of-grade study samples of 2003-04 North Carolina fourth graders linked to birth certificate data, by race group

\begin{tabular}{|c|c|c|c|c|c|c|}
\hline & \multicolumn{3}{|c|}{ Reading study sample } & \multicolumn{3}{|c|}{ Math study sample } \\
\hline & $\begin{array}{c}\text { Non-Hispanic } \\
\text { white } \\
\begin{array}{c}(n=43064) \\
n(\%)^{\mathrm{a}}\end{array}\end{array}$ & $\begin{array}{c}\text { Non-Hispanic } \\
\text { black } \\
\begin{array}{c}(n=21238) \\
n(\%)^{\mathrm{a}}\end{array}\end{array}$ & $P$-value ${ }^{\mathrm{b}}$ & $\begin{array}{c}\text { Non-Hispanic } \\
\text { white } \\
\begin{array}{c}(n=43165) \\
n(\%)^{\mathrm{a}}\end{array}\end{array}$ & $\begin{array}{c}\text { Non-Hispanic } \\
\text { black } \\
(n=21321) \\
n(\%)^{\mathrm{a}}\end{array}$ & $P$-value ${ }^{\mathrm{b}}$ \\
\hline Reading EOG test score, mean (SD) & $254(8)$ & $248(8)$ & $<0.001$ & & & \\
\hline Math EOG test score, mean (SD) & & & & $261(7)$ & $255(7)$ & $<0.001$ \\
\hline Gestational age, weeks, mean (SD) & $39(2)$ & $39(2)$ & $<0.001$ & $39(2)$ & $39(2)$ & $<0.001$ \\
\hline $\begin{array}{l}\text { Birthweight percentile for gestational age, } \\
\text { mean (SD) }\end{array}$ & $51(29)$ & $39(27)$ & $<0.001$ & $51(29)$ & $39(27)$ & $<0.001$ \\
\hline Gestational age, weeks & & & $<0.001$ & & & $<0.001$ \\
\hline$<34$ & $881(2)$ & $942(4)$ & & $882(2)$ & $949(4)$ & \\
\hline $34-36$ & $2531(6)$ & $1841(9)$ & & $2542(6)$ & $1850(9)$ & \\
\hline$\geq 37$ & $39652(92)$ & $18455(87)$ & & $39741(92)$ & $18522(87)$ & \\
\hline Small for gestational age & $3997(9)$ & $3526(17)$ & $<0.001$ & $4023(9)$ & $3546(17)$ & $<0.001$ \\
\hline Mother reported smoking during pregnancy & $9358(22)$ & $2573(12)$ & $<0.001$ & $9390(22)$ & $2590(12)$ & $<0.001$ \\
\hline Maternal age, years & & & $<0.001$ & & & $<0.001$ \\
\hline $15-19$ & $5497(13)$ & $5445(26)$ & & $5520(13)$ & $5464(26)$ & \\
\hline $20-24$ & $11725(27)$ & $7330(35)$ & & $11758(27)$ & $7353(34)$ & \\
\hline $25-29$ & $12257(28)$ & $4532(21)$ & & $12281(28)$ & $4550(21)$ & \\
\hline $30-34$ & $9755(23)$ & $2773(13)$ & & $9767(23)$ & $2792(13)$ & \\
\hline $35-39$ & $3426(8)$ & $1013(5)$ & & $3433(8)$ & $1015(5)$ & \\
\hline $40-44$ & $404(1)$ & $145(1)$ & & $406(1)$ & $147(1)$ & \\
\hline Maternal educational attainment level & & & $<0.001$ & & & $<0.001$ \\
\hline Less than high school & $8174(19)$ & $5765(27)$ & & $8208(19)$ & $5805(27)$ & \\
\hline High school diploma & $15957(37)$ & $9575(45)$ & & $16010(37)$ & $9608(45)$ & \\
\hline Some college & $9646(22)$ & $4196(20)$ & & $9659(22)$ & $4204(20)$ & \\
\hline College or more & $9287(22)$ & $1702(8)$ & & $9288(22)$ & $1704(8)$ & \\
\hline Mother unmarried at the time of delivery & $7927(18)$ & $14873(70)$ & $<0.001$ & $7956(18)$ & $14937(70)$ & $<0.001$ \\
\hline Child male sex & $21897(51)$ & $10610(50)$ & $<0.05$ & $21977(51)$ & $10684(50)$ & 0.056 \\
\hline $\begin{array}{l}\text { Child participation in school free or reduced } \\
\text { lunch programme }\end{array}$ & $12802(30)$ & $16073(76)$ & $<0.001$ & $12864(30)$ & $16152(76)$ & $<0.001$ \\
\hline
\end{tabular}

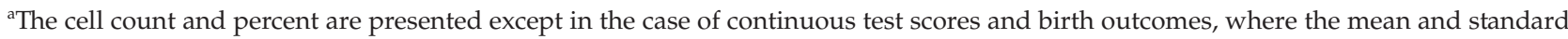
deviation are given as indicated next to the variable name.

${ }^{b}$ Chi-square test was used to test for differences by race group for categorical variables. ANOVA was used for continuous EOG test scores which were approximately normally distributed. Gestational age and birthweight percentile for gestational age were not normal. For these variables, the Kruskal-Wallis test was used to test for differences by race group.

smoking mothers had average test scores 1.25 points lower than NHB children of similar gestational age born to non-smoking mothers [marginal effect $=-1.25$, $95 \%$ CI $-2.47,-0.03]$. We did not observe a marginal effect of smoking among NHB children born between 34 and 36 weeks; however, among term birth, the marginal effect of smoking was -0.67 points $[95 \% \mathrm{CI}$ $-1.02,-0.32]$.

In the analysis of mathematics test scores, among NHW children, the marginal effect of smoking within gestational age category was similar to that of the reading analysis. In contrast, among NHB children, a marginal effect of smoking was observed only among late preterm and term births, with similar effect magnitudes of -0.88 [95\% CI $-1.64,-0.12]$ and -0.65 [95\% CI $-0.95,-0.35]$ points, respectively.

The relationships between reading and mathematics EOG test scores and continuous gestational age by maternal smoking status are depicted in Figures 1 and 2 , respectively. Predicted mean test scores according to gestational age are presented for non-smoking mothers (solid line) vs. smoking mothers (dotted line). Shading around each line represents the 95\% CI for the predicted scores. Vertical lines shown at 34 and 

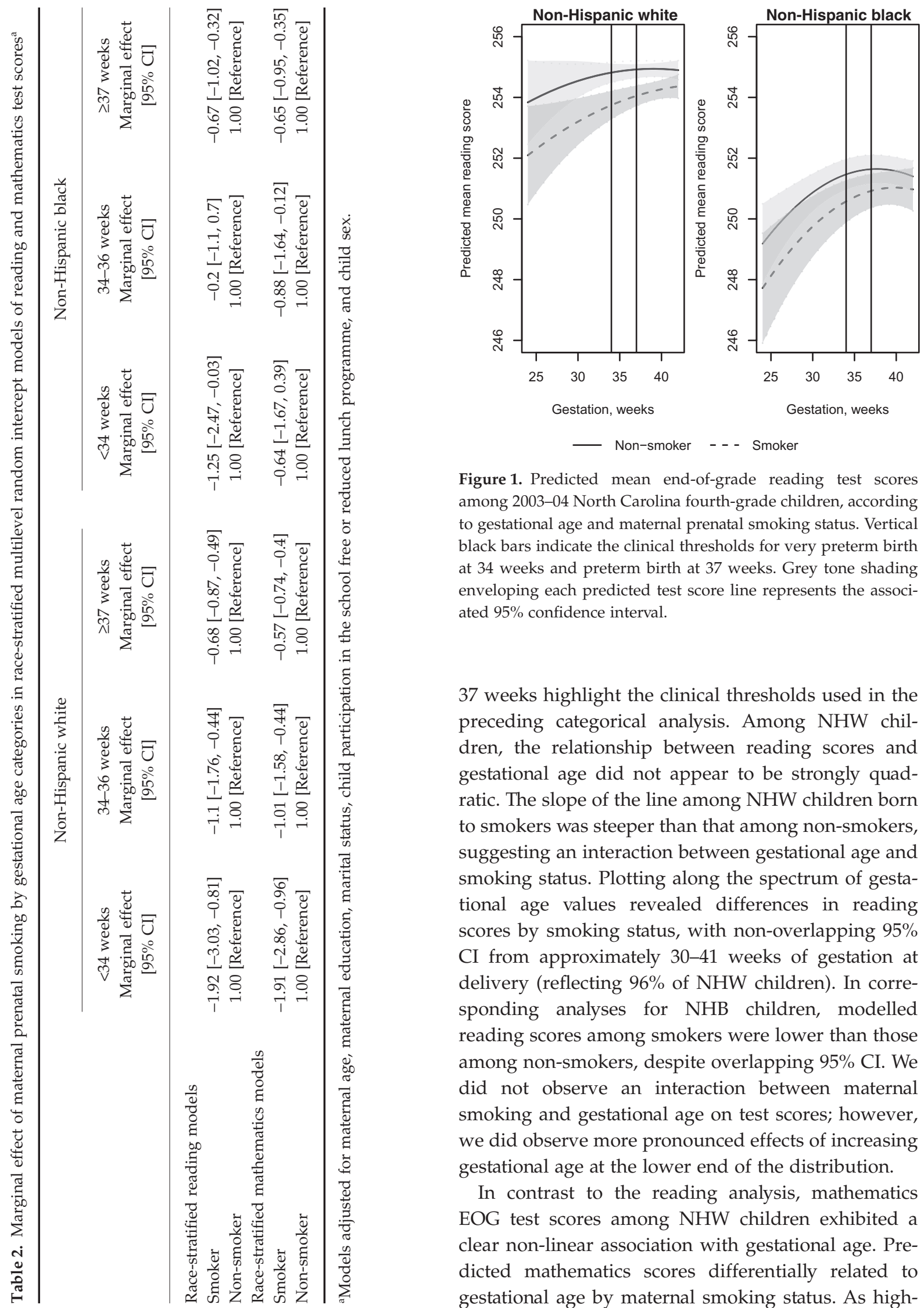

Figure 1. Predicted mean end-of-grade reading test scores among 2003-04 North Carolina fourth-grade children, according to gestational age and maternal prenatal smoking status. Vertical black bars indicate the clinical thresholds for very preterm birth at 34 weeks and preterm birth at 37 weeks. Grey tone shading enveloping each predicted test score line represents the associated $95 \%$ confidence interval.

37 weeks highlight the clinical thresholds used in the preceding categorical analysis. Among NHW children, the relationship between reading scores and gestational age did not appear to be strongly quadratic. The slope of the line among NHW children born to smokers was steeper than that among non-smokers, suggesting an interaction between gestational age and smoking status. Plotting along the spectrum of gestational age values revealed differences in reading scores by smoking status, with non-overlapping $95 \%$ CI from approximately 30-41 weeks of gestation at delivery (reflecting $96 \%$ of NHW children). In corresponding analyses for NHB children, modelled reading scores among smokers were lower than those among non-smokers, despite overlapping 95\% CI. We did not observe an interaction between maternal smoking and gestational age on test scores; however, we did observe more pronounced effects of increasing gestational age at the lower end of the distribution.

In contrast to the reading analysis, mathematics EOG test scores among NHW children exhibited a clear non-linear association with gestational age. Predicted mathematics scores differentially related to gestational age by maternal smoking status. As high- 


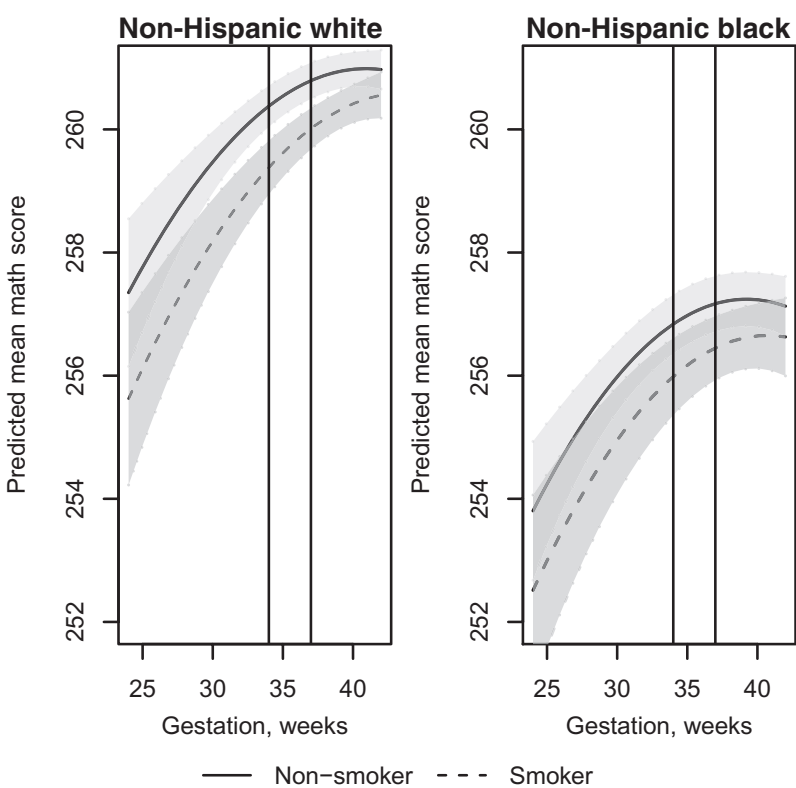

Figure 2. Predicted mean end-of-grade mathematics test scores among 2003-04 North Carolina fourth-grade children, according to gestational age and maternal prenatal smoking status. Vertical black bars indicate the clinical thresholds for very preterm birth at 34 weeks and preterm birth at 37 weeks. Grey tone shading enveloping each predicted test score line represents the associated $95 \%$ confidence interval.

lighted by the non-overlapping 95\% CI for the nonsmoking and smoking groups, this was particularly true in the range of 29-39 weeks gestational age, representing $47 \%$ of NHW children. Among NHB children, maternal smoking was associated with a downward shift in predicted mathematics scores; however, 95\% CI overlapped along the entire gesta- tional age distribution. The slopes of the curves of predicted test scores according to gestational age were similar by smoking status. The association between mathematics scores and gestational age appeared quadratic, with a diminishing effect of gestational age at the upper end of the distribution.

\section{Fetal growth}

Marginal effects of smoking during pregnancy by small for gestational age status are shown in Table 3. In both the reading and mathematics models, NHW children born to smoking mothers experienced a decrease in test scores that was similar by small for gestational age status. In contrast, among NHB children, for both reading and mathematics test scores, we observed a marginal effect of smoking equal to an approximate 0.7 point decrease only within non-small for gestational age births. In the mathematics model, an NHB child born small for gestational age to a smoking mother had an average mathematics score 0.51 points lower than a NHB child similarly born but to a non-smoking mother; however, this effect was characterised by a slightly wide $95 \%$ CI $[-1.05,0.03]$.

Predicted mean reading and mathematics EOG test scores according to birthweight percentile for gestational age are shown in Figures 3 and 4, respectively. The solid line represents non-smoking mothers, while the dotted line, smoking mothers. Shading around each line again corresponds to the $95 \%$ CI for the predicted scores. A vertical line at the 10th percentile shows the clinical threshold for small for gestational

Table 3. Marginal effect of maternal prenatal smoking by small for gestational age status in race-stratified multilevel random intercept models of reading and mathematics test scores ${ }^{a}$

\begin{tabular}{|c|c|c|c|c|}
\hline & \multicolumn{2}{|c|}{ Non-Hispanic white } & \multicolumn{2}{|c|}{ Non-Hispanic black } \\
\hline & $\begin{array}{c}\text { Small for } \\
\text { gestational age } \\
\text { Marginal effect } \\
{[95 \% \text { CI }]}\end{array}$ & $\begin{array}{c}\text { Non-small for } \\
\text { gestational age } \\
\text { Marginal effect } \\
{[95 \% \mathrm{CI}]}\end{array}$ & $\begin{array}{c}\text { Small for } \\
\text { gestational age } \\
\text { Marginal effect } \\
{[95 \% \text { CI }]}\end{array}$ & $\begin{array}{c}\text { Non-small for } \\
\text { gestational age } \\
\text { Marginal effect } \\
{[95 \% \mathrm{CI}]}\end{array}$ \\
\hline \multicolumn{5}{|c|}{ Race-stratified reading models } \\
\hline Smoker & $-0.68[-1.15,-0.21]$ & $-0.66[-0.86,-0.46]$ & $-0.44[-1.08,0.2]$ & $-0.7[-1.07,-0.33]$ \\
\hline Non-smoker & 1.00 [Reference] & 1.00 [Reference] & 1.00 [Reference] & 1.00 [Reference] \\
\hline \multicolumn{5}{|c|}{ Race-stratified mathematics models } \\
\hline Smoker & $-0.65[-1.06,-0.24]$ & $-0.55[-0.72,-0.38]$ & $-0.51[-1.05,0.03]$ & $-0.71[-1.02,-0.4]$ \\
\hline Non-smoker & 1.00 [Reference] & 1.00 [Reference] & 1.00 [Reference] & 1.00 [Reference] \\
\hline
\end{tabular}

${ }^{a}$ Models adjusted for maternal age, maternal education, marital status, child participation in the school free or reduced lunch programme, and child sex. 


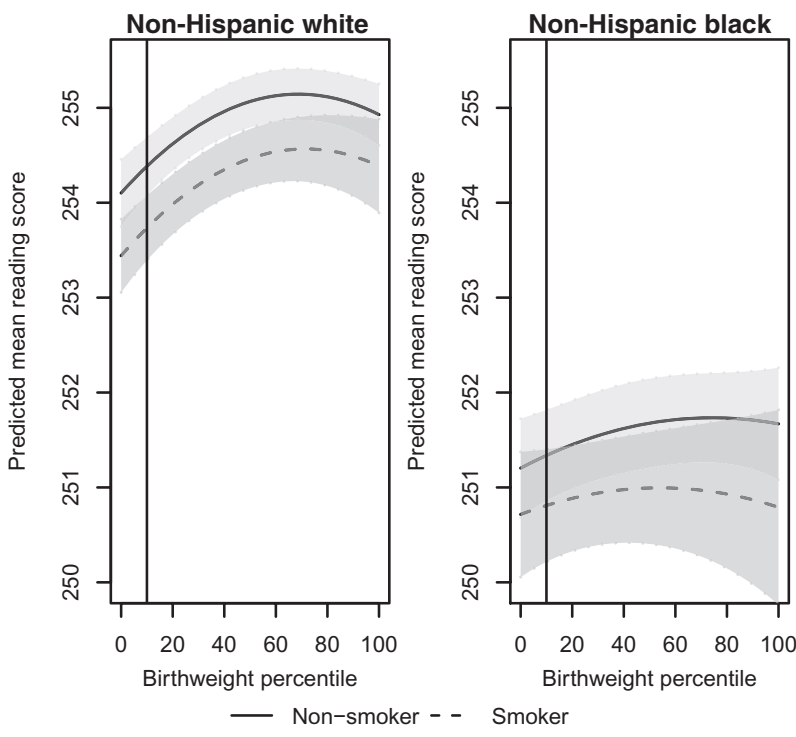

Figure 3. Predicted mean end-of-grade reading test scores among 2003-04 North Carolina fourth-grade children, according to birthweight percentile for gestational age and maternal prenatal smoking status. The vertical black bar indicates the clinical threshold for small for gestational age at the 10th percentile. Grey tone shading enveloping each predicted test score line represents the associated $95 \%$ confidence interval.

age. With the exception of the reading model for NHB children, reading and mathematics test scores were quadratically associated with birthweight percentile for gestational age. Maternal smoking was associated with a downward shift in test scores across the range of birthweight percentile for gestational age, especially so from the $11^{\text {th }}$ to $58^{\text {th }}$ percentile in the NHW reading analysis. This range represents approximately $46 \%$ of births, all of which meet the clinical standard for fetal growth of above the 10th birthweight percentile for gestational age. In the NHB analysis, the $95 \%$ CI for predicted reading and mathematics test scores according to birthweight percentile for gestational age by maternal smoking were overlapping. The difference between predicted reading scores by smoking widened with increasing birthweight percentile for gestational age.

\section{Model covariates}

Covariates behaved similarly across all models (see online supporting information). Participation in the school free or reduced lunch programme, if a mother was unmarried, and if she had less than a high school education, were negatively related to EOG test performance. Across models, holding a given birth outcome at its sample mean, a child whose mother smoked during pregnancy had, on average, lower mean reading and mathematics EOG test scores than a child whose mother did not smoke during pregnancy, with the magnitude of the coefficients representing $5-7 \%$ of the interquartile range (IQR) of test scores for both subjects. (For the reading analysis, the IQR was 11 and 12 points for NHW children and NHB children, respectively. The corresponding figures for the mathematics analysis were 10 and 9 points.) Estimated between-school variability was larger among NHB test scores than NHW test scores regardless of subject matter.

\section{Comment}

Using population-level birth and educational data from NC, we examined how maternal prenatal smoking impacts the associations between birth outcomes and EOG reading and mathematics scores at fourth grade. Our findings highlight five main themes, which we comment on below.

First, among both NHW and NHB children, maternal prenatal smoking exhibited a negative association
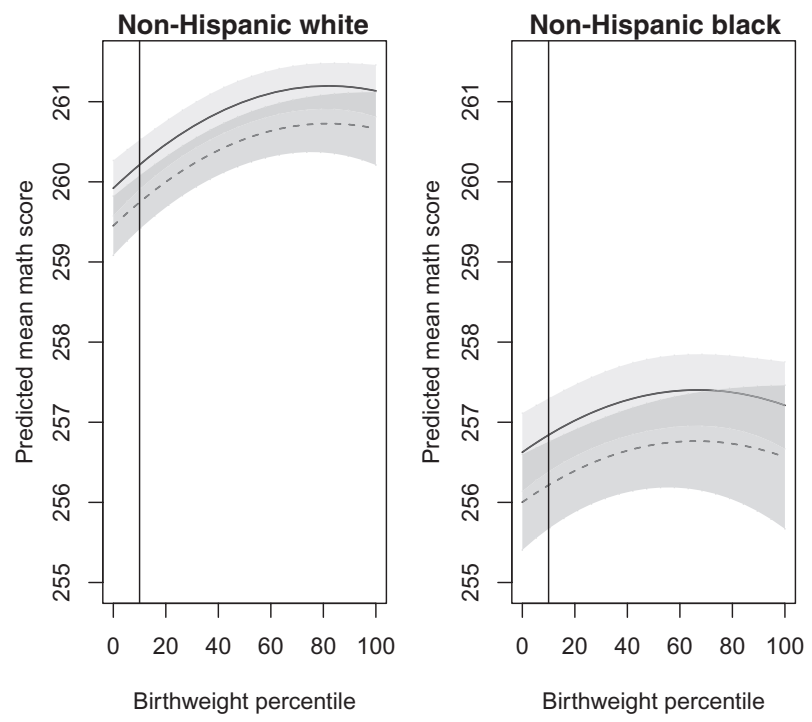

Figure 4. Predicted mean end-of-grade mathematics test scores among 2003-04 North Carolina fourth-grade children, according to birthweight percentile for gestational age and maternal prenatal smoking status. The vertical black bar indicates the clinical threshold for small for gestational age at the 10th percentile. Grey tone shading enveloping each predicted test score line represents the associated $95 \%$ confidence interval. 
with EOG test scores, even within clinically healthy term births and non-small for gestational age births. This highlights a significant number of children, many of whom did not experience an adverse birth outcome, for whom maternal smoking behaviour during pregnancy may still be relevant for academic achievement. These findings reaffirm the importance of reducing maternal prenatal smoking rates.

Second, irrespective of race group, a change in continuous gestational age or birthweight percentile for gestational age had a greater impact at the lower versus the normal or upper range of each birth outcome.

Third, we found important differences in the relationship among birth outcomes, maternal prenatal smoking, and educational achievement by race. Among NHW children, the slope of the relationship between predicted test scores and birth outcomes was often steeper among children whose mothers smoked during pregnancy than in the non-smoking group; thus, the difference in test scores between nonsmokers and smokers diminished with increasingly better birth outcomes. In contrast, improvements in birth outcome among NHB children did not diminish the gap in test scores between children of nonsmokers and smokers. We hypothesise that these findings may be rooted in the disproportionate, concentrated social, economic, and environmental disadvantage that often confronts NHB children in the US. This does not, however, negate the importance of smoking avoidance and cessation programmes for women of all race groups, as the predicted test scores among NHB children born to smoking mothers were invariably lower. Maternal smoking behaviour during and after pregnancy has important consequences on children's health. ${ }^{28-30}$ In addition, smoking is an actionable health behaviour that can be addressed at individual and community levels.

Fourth, our findings highlight improvements in test scores, particularly for mathematics, associated with improvements in birth outcomes even within clinically defined normal ranges, although this pattern was less pronounced among NHB than NHW children. This is consistent with previous studies that have considered birth outcomes extending into the normal range. ${ }^{5-9,31}$

Fifth, categorising birth outcomes according to clinical thresholds can make analytic results easily translatable to clinical interpretation and understanding; however, our results highlight that continuous measures of birth outcomes provide additional insights regarding the complex associations among birth outcomes, maternal prenatal smoking, and academic performance. Some insights were provided by analyses using either categorical or continuous measures of birth outcomes. For example, an interaction between gestational age and maternal smoking was observed in the NHW analysis whether gestational age was operationalised as a categorical or continuous measure. However, using only clinical threshold categories, which grouped all births occurring in the normal range of the birth outcomes, would have prevented us from observing the nature of the relationship between test scores and birth outcomes within the normal range, which we found to be non-constant. The non-linear relationships between birth outcomes and test scores were also not as clearly characterised when we categorised the birth outcomes. Using continuous birth outcomes, when available, may help clarify our understanding of the complex associations among birth outcomes, maternal prenatal smoking, and academic performance.

While changes in EOG scores associated with maternal prenatal smoking or differences in birth outcomes, especially within the normal range, are only estimated to be a few points, even such small changes can have important long-term implications. Success on reading and mathematics achievement tests is an important predictor of long-term economic success. ${ }^{32}$ EOG test scores are often used to make key decisions about children's educational paths. For children already on the lower end of the test score distribution, a reduction in EOG test scores associated with a smaller birthweight percentile or lower gestational age may lead to the child missing the cut-off for promotion to the next grade. In our reading analysis dataset, 729 NHW children $(1.7 \%)$ were one point away from receiving the minimum passing reading test score, while $1432(3.3 \%)$ were within 2 points away from passing. Among NHB children, these figures were $878(4.1 \%)$ and $1699(8 \%)$, respectively. In the context of the estimated marginal effects that ranged from just over half a point to nearly 2 points, reducing smoking rates within very preterm, late preterm, and term births can have real consequences for reading performance. This can have long-term consequences, as grade retention has been linked with lower rates of high school graduation ${ }^{33}$ and participation in higher education programmes. ${ }^{34}$ For children on the other end of the scale, an increase in EOG test scores associated with an improvement in birth 
outcomes or a mother quitting smoking during pregnancy may permit entry into accelerated or gifted education programmes, offering numerous academic opportunities not available to all students.

This study is not without limitations. The prenatal smoking data in the NCDBR are self-reported and may not accurately represent smoking patterns during pregnancy; however, previous work has determined self-reported smoking information in the NCDBR to be reasonably well reported. ${ }^{23}$ Moreover, under the scenario of reporting inaccuracy, maternal smoking during pregnancy is likely to be systematically underreported, resulting in our underestimating the absolute magnitude of its association with EOG test scores. Also, measuring prenatal smoking as a binary indicator, as opposed to using the reported number of cigarettes smoked daily, may mask the nuanced nature of the relationship with test scores (e.g. potential threshold effects). In this way, our estimated coefficient on smoking represents an average over the range of possible smoking intensities. Last, with regard to our smoking exposure, we do not have any data on postnatal exposure to environmental tobacco smoke, which has been associated with cognitive deficits in children. ${ }^{35}$ We posit, however, that women who smoke during pregnancy are unlikely to quit after giving birth. Thus, the estimated coefficient on maternal prenatal smoking in our analysis may also be picking up the effect of childhood exposure to cigarette smoke between birth and test day. Our findings are also subject to bias from potential unmeasured confounders, particularly those related to possible endogeneity associated with maternal prenatal smoking, like the quality of the home environment or maternal IQ. Such factors may determine both child test performance and the likelihood that a mother smokes during pregnancy. We attempted to mitigate this bias by controlling for maternal educational attainment and participation in the school free or reduced lunch programme, in addition to including school and school district random effects to capture unobserved social and economic factors at these aggregate levels.

Despite its limitations, this study enriches the existing literature on the link between in utero exposures/ pregnancy outcomes and cognitive outcomes. Our emphasis on a population-level analysis of children who are roughly the same age (fourth graders) using achievement (rather than aptitude) outcomes complements the important work in this area by previous researchers. ${ }^{1,25,16}$ This work adds to the more limited literature that includes children born across the full range of birth outcomes, ${ }^{5-7}$ and our exploration of non-linear associations offers additional flexibility to our understanding of the association between birth outcomes and children's cognitive development. By conducting this analysis in a race-stratified setting, we offer an exploration of how these factors may contribute to and mitigate against educational outcome disparities in a population where adverse birth outcomes are more common among NHB children, ${ }^{36}$ and smoking is more common among NHW women. ${ }^{37}$

More work is needed to fully disentangle the complex relationships among birth outcomes, maternal prenatal smoking, and educational outcomes, including formal mediation analysis and quantile regression exploring how exposures may have different impacts along the distribution of test scores. It is clear, however, that pregnancy-related exposures and outcomes have a significant and long-term impact on child development. Clinically, this work affirms the importance of physicians engaging paediatric and adult patients regarding smoking cessation programmes. Given the beneficial impact on academic outcomes of improved birth outcomes even within the healthy range, this work emphasises the importance of creating protective environments in utero and achieving better outcomes for pregnant mothers.

\section{Acknowledgements}

This work was supported by the US Environmental Protection Agency [grant number RD-83329301]. We gratefully acknowledge data management support provided by Claire Osgood.

\section{References}

1 Aarnoudse-Moens CS, Weisglas-Kuperus N, van Goudoever JB, Oosterlaan J. Meta-analysis of neurobehavioral outcomes in very preterm and/or very low birth weight children. Pediatrics 2009; 124:717-728.

2 de Rodrigues MCC, Mello RR, Fonseca SC. Learning disorders, very low birth weight, systematic review. Jornal de Pediatria 2005; 82:6-14.

3 Marlow N, Wolke D, Bracewell MA, Samara M. Neurologic and developmental disability at six years of age after extremely preterm birth. New England Journal of Medicine 2005; 352:9-19.

4 Kerr-Wilson CO, Mackay DF, Smith GC, Pell JP. Meta-analysis of the association between preterm delivery and intelligence. Journal of Public Health (Oxford, England) 2012; 34:209-216. 
5 Kirkegaard I, Obel C, Hedegaard M, Henriksen TB. Gestational age and birth weight in relation to school performance of 10-year-old children: a follow-up study of children born after 32 completed weeks. Pediatrics 2006; 118:1600-1606.

6 Rahu K, Rahu M, Pullmann H, Allik J. Effect of birth weight, maternal education and prenatal smoking on offspring intelligence at school age. Early Human Development 2010; 86:493-497.

7 Matte TD, Bresnahan M, Begg MD, Susser E. Influence of variation in birth weight within normal range and within sibships on IQ at age 7 years: cohort study. British Medical Journal 2001; 323:310-314.

8 Noble KG, Fifer WP, Rauh VA, Nomura Y, Andrews HF. Academic achievement varies with gestational age among children born at term. Pediatrics 2012; 130:e257-e264.

9 Lipkind HS, Slopen ME, Pfeiffer MR, McVeigh KH. School-age outcomes of late preterm infants in New York City. American Journal of Obstetrics and Gynecology 2012; 206:222-226.

10 Mackay DF, Smith GC, Dobbie R, Pell JP. Gestational age at delivery and special educational need: retrospective cohort study of 407,503 schoolchildren. PLoS Medicine 2010; 7(6):e1000289.

11 Yang S, Platt RW, Kramer MS. Variation in child cognitive ability by week of gestation among healthy term births. American Journal of Epidemiology 2010; 171:399-406.

12 DiFranza JR, Aligne CA, Weitzman M. Prenatal and postnatal environmental tobacco smoke exposure and children's health. Pediatrics 2004; 113:1007-1015.

13 Stillerman KP, Mattison DR, Giudice LC, Woodruff TJ. Environmental exposures and adverse pregnancy outcomes: a review of the science. Reproduction Science 2008; 15:631-650.

14 Weitzman M, Byrd RS, Aligne CA, Moss M. The effects of tobacco exposure on children's behavioral and cognitive functioning: implications for clinical and public health policy and future research. Neurotoxicology and Teratology 2002; 24:397-406.

15 Julvez J, Ribas-Fito N, Torrent M, Forns M, Garcia-Esteban $\mathrm{R}$, Sunyer J. Maternal smoking habits and cognitive development of children at age 4 years in a population-based birth cohort. International Journal of Epidemiology 2007; 36:825-832.

$16 \mathrm{O}^{\prime}$ Callaghan FV, Al MA, O'Callaghan M, Alati R, Williams GM, Najman JM. Is smoking in pregnancy an independent predictor of academic difficulties at 14years of age? A birth cohort study. Early Human Development 2010; 86:71-76.

17 Mortensen EL, Michaelsen KF, Sanders SA, Reinisch JM. A dose-response relationship between maternal smoking during late pregnancy and adult intelligence in male offspring. Paediatric and Perinatal Epidemiology 2005; 19:4-11.

18 Cho K, Frijters JC, Zhang H, Miller LL, Gruen JR. Prenatal exposure to nicotine and impaired reading performance. The Journal of Pediatrics 2012; 162:713-718.

19 Batty GD, Der G, Deary IJ. Effect of maternal smoking during pregnancy on offspring's cognitive ability: empirical evidence for complete confounding in the US national longitudinal survey of youth. Pediatrics 2006; 118:943-950.

20 Breslau N, Paneth N, Lucia VC, Paneth-Pollak R. Maternal smoking during pregnancy and offspring IQ. International Journal of Epidemiology 2005; 34:1047-1053.

21 Gilman SE, Gardener H, Buka SL. Maternal smoking during pregnancy and children's cognitive and physical development: a causal risk factor? American Journal of Epidemiology 2008; 168:522-531.

22 Buescher PA, Taylor KP, Davis MH, Bowling JM. The quality of the new birth certificate data: a validation study in North Carolina. American Journal of Public Health 1993; 83:1163-1165.

23 Buescher P. Smoking in pregnancy in North Carolina. North Carolina Medical Journal 1997; 58:356-360.

24 North Carolina Public Schools. Understanding North Carolina end-of-grade testing. Assessment Brief 2004; 5:1-3.

25 North Carolina Public Schools Accountability Services Division. The North Carolina testing program 2006-2007. North Carolina Department of Public Instruction: Raleigh, North Carolina. 2006. 12-13-2006.

26 Miranda ML, Messer LC, Kroeger GL. Associations between the quality of the residential built environment and pregnancy outcomes among women in North Carolina. Environmental Health Perspectives 2012; 120:471-477.

27 Oehlert GW. A note on the delta method. The American Statistician 1992; 46:27-29.

28 Burke H, Leonardi-Bee J, Hashim A, Pine-Abata H, Chen Y, Cook DG, et al. Prenatal and passive smoke exposure and incidence of asthma and wheeze: systematic review and meta-analysis. Pediatrics 2012; 129:735-744.

29 Ino T. Maternal smoking during pregnancy and offspring obesity: meta-analysis. Pediatrics International: Official Journal of the Japan Pediatric Society 2010; 52:94-99.

30 Jones LL, Hassanien A, Cook DG, Britton J, Leonardi-Bee J. Parental smoking and the risk of middle ear disease in children: a systematic review and meta-analysis. Archives of Pediatrics \& Adolescent Medicine 2012; 166:18-27.

31 Moster D, Lie RT, Markestad T. Long-term medical and social consequences of preterm birth. New England Journal of Medicine 2008; 359:262-273.

32 Currie J, Thomas D. Early test scores, school quality and SES: Longrun effects on wage and employment outcomes. Research in Labor Economics 2001; 20:103-132.

33 Jimerson SR. Meta-analysis of grade retention research: implications for practice in the 21st century. School Psychology Review 2001; 30:420-437.

$34 \mathrm{Ou} \mathrm{S}$, Reynolds AJ. Grade retention, postsecondary education, and public aid receipt. Educational Evaluation and Policy Analysis 2010; 32:118-139.

35 Yolton K, Dietrich K, Auinger P, Lanphear BP, Hornung R. Exposure to environmental tobacco smoke and cognitive abilities among U.S. children and adolescents. Environmental Health Perspectives 2005; 113:98-103.

36 Miranda ML, Maxson P, Edwards S. Environmental contributions to disparities in pregnancy outcomes. Epidemiologic Reviews 2009; 31:67-83. 
37 U.S. Centers for Disease Control and Prevention. Trends in smoking before, during, and after pregnancy - Pregnancy Risk Assessment Monitoring System (PRAMS), United States, 31 sites, 2000-2005. MMWR. Morbidity and Mortality Weekly Report 2009; 58:1-29.

\section{Supporting information}

Additional Supporting Information may be found in the online version of this article at the publisher's web-site:

Table S1. Race-stratified multilevel random intercept models of the relationship between reading and mathematics EOG test scores and gestational age categories and maternal smoking status among 2003-04 NC fourth graders linked to birth certificate data.
Table S2. Race-stratified multilevel random intercept models of the relationship between reading and mathematics EOG test scores and small for gestational age and maternal smoking status among 2003-04 NC fourth graders linked to birth certificate data.

Table S3. Race-stratified multilevel random intercept models of the relationship between reading and mathematics EOG test scores and gestational age and maternal smoking status among 2003-04 NC fourth graders linked to birth certificate data.

Table S4. Race-stratified multilevel random intercept models of the relationship between reading and mathematics EOG test scores and birthweight percentile for gestational age and maternal smoking status among 2003-04 NC fourth graders linked to birth certificate data. 